\title{
Online KVT-I: eine eierlegende Wollmilchsau?
}

Fragestellung: Kann bei Studenten mit insomnischer Störung durch eine psychotherapeutische Behandlung der Schlafstörung mithilfe eines Onlineprogramms auch eine Reduktion psychotischer Symptome erzielt werden?

Hintergrund: Forschungen der letzten Jahre zeigen, dass das Vorliegen einer insomnischen Störung (Ein- oder Durchschlafstörungen mit Beeinträchtigung der Tagesbefindlichkeit) das Risiko für die Neuerkrankung an einer psychischen Störung erhöht. Insomnische Störungen können mit kognitiver Verhaltenstherapie für Insomnie (KVT-I) gut behandelt werden. Es scheint daher naheliegend, die Behandlung insomnischer Störungen zur Prävention psychischer Erkrankungen zu nutzen. Einige Studien konnten zeigen, dass sich nach KVT-I auch über die Schlafstörung hinausgehende psychische Beschwerden bessern. Insgesamt ist die Datenlage zum Thema Prävention jedoch noch dünn.

Freeman D, Sheaves B, Goodwin $\mathrm{G}$ et al. The effects of improving sleep on mental health (OASIS): a randomised controlled trial with mediation analysis. Lancet Psychiatry 2017: 4; 749-58
Patienten und Methodik: In die Studie wurden 3.755 Studenten mit insomnischer Störung von 26 Universitäten in Großbritannien eingeschlossen. Bis auf ein positives Insomnie-Screening mit- tels eines Fragebogens und ein Alter über 18 Jahre bestanden keine Ein- oder Ausschlusskriterien. Die Teilnehmer wurden randomisiert entweder einem Online-Therapieprogramm für insomnische Störungen (Sleepio) oder Treatment as Usual (TAU) zugeordnet. Das Sleepio-Programm beinhaltet sechs Sitzungen à zirka 20 Minuten, die online über ein Smartphone zugänglich sind. Hauptoutcomeparameter waren drei Fragebögen, die den Schweregrad der insomnischen Störung (Sleep Condition Indicator), paranoide Symptome (Green Paranoid Thought Scales) und halluzinatorische Symptome (Specific Psychotic Experiences Questionnaire - Hallucinations subscale) messen.

Ergebnisse: Am Behandlungsende und zwölf Wochen später zeigte die Sleepio-Gruppe eine signifikant stärkere Reduktion insomnischer Symptome sowie paranoider und halluzinatorischer Symptome als die TAU-Gruppe. Die Reduktion insomnischer Symptome war ein Mediator bezüglich der Reduktion psychotischer Symptome.

Schlussfolgerungen: Die Autoren folgern, dass Insomnie ein kausaler Faktor in der Entstehung psychotischer Erfahrungen und anderer psychischer Probleme ist. Der Behandlung insomnischer Störungen sollte daher innerhalb des Gesundheitssystems eine höhere Priorität eingeräumt werden.

\section{- Kommentar von Dieter Riemann und Elisabeth Hertenstein, Freiburg i. Br}

\section{Kausalität differenzierter hinterfragen}

Die Daten zeigen eindrucksvoll, dass eine online durchgeführte KVT-I geeignet ist, insomnische Störungen in einer großen Stichprobe effektiv und mit vergleichsweise geringem Ressourcenaufwand zu behandeln. Hauptfokus der vorliegenden Studie war aber nicht die Insomniebehandlung, sondern die Prävention psychotischer Erkrankungen. Die Autoren folgern aus ihren Daten, dass insomnische Störungen eine kausale Rolle in der Entstehung psychotischer Störungen spielen. Hier muss jedoch einschränkend erwähnt werden, dass es sich bei der untersuchten Stichprobe um Studenten mit Schlafstörungen handelte. Das Vorliegen relevanter psychotischer Symptome war kein Einschlusskritierum, auch wurde nicht erfasst, bei wie vielen Teilnehmern tatsächlich eine psychotische Störung vorlag. Bekannten Prävalenzzahlen zufolge dürfte es sich schätzungsweise um 1-2\% handeln. Während die per Fragebogen erfassten psychotischen Erfahrungen in der Gesamtstichprobe reduziert werden konnten, bleibt unklar, wie sich dies in der Substichprobe der Teilnehmer mit tatsächlich bestehender psychotischer Störung verhielt. Bei einem Messzeitraum von nur zwölf Wochen kann kaum beurteilt werden, inwiefern das Therapieprogramm zur Prävention psychotischer Erkrankungen beitrug. Zudem stellt sich die Frage, was genau ein Fragebogen zu psychotischem Erleben bei Teilnehmern ohne tatsächliche psychotische Störung überhaupt misst. Auf der "Green Paranoid
Thought Scale" geben die Teilnehmer beispielsweise an, inwiefern sie in der letzten Zeit das Gefühl hatten, von anderen kritisch bewertet oder ausgelacht zu werden. Ohne ausführliche Anamnese kann nicht abschließend geklärt werden, ob es sich hierbei tatsächlich um psychotisches Erleben handelt. Eine Alternativerklärung für den Rückgang der Werte auf der genannten Skala sind Verbesserungen von Allgemeinbefinden, Selbstwert und Lebensqualität durch die Insomniebehandlung.

Zusammenfassend handelt es sich um eine gut durchdachte, stringent durchgeführte Studie, die die Relevanz digitaler Verhaltenstherapie für die Therapie insomnischer Störungen untermauert. Die Schlussfolgerungen bezüglich der Kausalität psychotischer Erkrankungen sind jedoch mit Vorsicht zu bewerten.

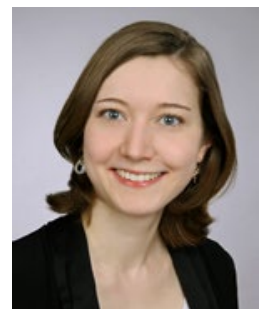

Dr. Elisabeth Hertenstein und Prof. Dr. Dieter Riemann

Klinik für Psychiatrie und Psychotherapie Universitätsklinikum Freiburg E-Mail: dieter.riemann@uniklinik-freiburg.de elisabeth.hertenstein@uniklinik-freiburg.de 